\section{Transcatheter mitral valve-in-valve implantation in patients with degenerated bio-prostheses - Experience of a Centre in Mexico}

\section{Implantación valvular mitral transcatéter \\ "valve-in-valve" en pacientes con bio-prótesis degeneradas - Experiencia de un centro en México}

Transcatheter "valve-in-valve" (VIV) implantation into a deteriorated mitral bioprosthesis is an uncommon technique worldwide. Reintervention of a mitral degenerated valve is associated with an elevated surgical risk, especially in elderly patients with multiple comorbidities. This technique provides an alternative to conventional surgery that avoids myocardial dissection, extracorporeal circulation, and myocardial ischemia. ${ }^{1}$ Most prostheses are implanted via a transapical approach; however, another route to access the degenerated prostheses is through the femoral vein, followed by perforation of the interatrial septum and anterograde valve implantation. ${ }^{2}$ Herein we present the first experience with this technique performed in our facilities and for our concern the first in Latin America.

From January to December 2016, 3 patients (age $74 \pm 12$ years) were admitted in our institution with signs of valve dysfunction long term after bio-prosthetic mitral valve replacement (MVR), 2 patients received transseptal implantation and 1 patient transapical implantation of a balloon-expandable pericardial heart valve into a degenerated bioprosthesis (range 24-29 mm) in mitral position at our institution. All patients were considered in high risk for surgical valve replacement (EuroSCORE II 15.755.75 $\pm 7.6 \%$, STS PROM [Society of Thoracic Surgeons predicted risk of mortality] $14.69 \pm 4.8 \%$ ) (Table 1 ) after the Heart Team evaluation, therefore were eligible for a VIV procedure via a transseptal or transapical approach.

Implantation was successful in all patients, 2 patients received transseptal implantation with an Edwards Sapien XT (Edwards Lifesciences, Irvine, California) valve (\#23 and \#29) and 1 patient transapical implantation with an Edwards Sapien (Edwards Lifesciences, Irvine, California) \#26 valve into a degenerated bioprosthesis (range 24-29 mm) in mitral position. Ecocardiography showed a reduction of mean transvalvular gradients from $18.5 \pm 9.5 \mathrm{~mm} \mathrm{Hg}$ to $3 \pm 1 \mathrm{~mm} \mathrm{Hg}$, with no paravalvular regurgitation remaining, the systolic pulmonary artery pressure (SPAP) had a reduction from $92.5 \pm 12.5 \mathrm{~mm} \mathrm{Hg}$ to $50 \pm 10 \mathrm{~mm} \mathrm{Hg}$ (Table 2). One patient with the transseptal approach developed a hospital acquired pneumonia and sepsis, then evolves into a septic shock and died 10 days after the procedure. In the remaining patients the New York Heart Association (NYHA) functional class improved from 3.0 to 1.0 , over a mean follow-up of 227 (IQR: 90-365) days.

Although the amount of patients reported is small, it exhibits that transapical and transseptal mitral VIV implantation can be feasible in high surgical risk patients, and showed favorable clinical and hemodynamic results in short and medium-term follow-up with low morbidity and low mortality. All patients improved NYHA functional class and had an important reduction in the transvalvular gradients and SPAP however; one of the patients died, cannot be attributed to the procedure.

Among the first multicenter registries: Webb et al. described the results of 23 consecutive patients successfully treated with transcatheter valve implantation through a transapical approach. The success rate of the device was $100 \%$. There was no intra-procedural or 30-day mortality. With a median follow-up of 753 days, the survival rate was 90.4\%. Clinical improvement in NYHA class I/II heart failure symptoms was observed in all patients except one (95.6\%). ${ }^{3}$

Favorable results have been reported in cases of implantation through a transseptal approach using the Sapien XT (Edwards Lifesciences, Irvine, California) valve, ${ }^{4}$ and the Melody (Medtronic, Santa Rosa, California) valve va,6 $^{5,}$ although the great majority of successful cases have used a transapical approach. ${ }^{3,7-9}$ The Dvir group reported results on 70 patients enrolled in the global VIVID registry $(11.4 \%$ valvein-ring, $88.6 \%$ valve-in-valve). All patients were treated with Sapien (Edwards Lifesciences, Irvine, California) valve (23 mm 22.9\%, $26 \mathrm{~mm} \mathrm{58.6 \% ,} \mathrm{and} 29 \mathrm{~mm} \mathrm{18.6 \% ).} \mathrm{Transapical}$ access was used in $85.7 \%$, transeptal in $10 \%$ and transatrial in 4.3\%. Malposition of the device occurred at $4.3 \%$. The 30 -day all-cause mortality rate was $10.3 \%$ and $82.3 \%$ of the patients remained in the $1-\|$ functional class at 30 days. ${ }^{10}$ The transseptal approach can be feasible in selected patients if the transapical approach is not possible for anatomic reasons or due to the surgical experience in the center.

\section{Financing}

The authors did not receive any payment or economic benefit for the preparation and execution of the article.

\section{Conflict of interests}

The authors declare do not have conflict of interest. 
Table 1 Baseline clinical parameters.

\begin{tabular}{|c|c|c|c|c|c|c|c|c|c|c|c|}
\hline \multicolumn{2}{|c|}{ Patient \#Age } & \multirow{2}{*}{$\begin{array}{l}\text { Euroscore II } \\
23.4\end{array}$} & \multirow{2}{*}{$\begin{array}{l}\text { STS-PROM } \\
19.56\end{array}$} & \multirow{2}{*}{$\begin{array}{l}\text { NYHA } \\
\text { functional class } \\
3\end{array}$} & \multirow{2}{*}{$\begin{array}{l}\text { Procedure } \\
\text { Transpical }\end{array}$} & \multirow{2}{*}{$\begin{array}{l}\text { Comorbidities } \\
\text { AF, COPD, } \\
\text { hypercholes- } \\
\text { terolemia }\end{array}$} & \multirow{2}{*}{$\begin{array}{l}\begin{array}{l}\text { Previous } \\
\text { sternotomies }\end{array} \\
1: \text { MVR, AVR }\end{array}$} & \multirow{2}{*}{$\begin{array}{l}\begin{array}{l}\text { Creatinine } \\
(\mathrm{mg} / \mathrm{dL})\end{array} \\
0.96\end{array}$} & \multirow{2}{*}{$\begin{array}{l}\text { SPAP }(\mathrm{mm} \mathrm{Hg}) \\
105\end{array}$} & \multirow{2}{*}{$\begin{array}{l}\text { TR grade } \\
3\end{array}$} & \multirow{2}{*}{$\begin{array}{l}\mathrm{EF}(\%) \\
76\end{array}$} \\
\hline 1 & 85 & & & & & & & & & & \\
\hline 2 & 78 & 8.2 & 9.82 & 3 & Transseptal & $\begin{array}{l}\text { IDDM, } \\
\text { hypertension, } \\
\text { AF, } \\
\text { decompensated } \\
\text { HF }\end{array}$ & 2: MVRep, MVR & 1.2 & 70 & 3 & 72 \\
\hline 3 & 62 & 8.1 & 12.2 & 3 & Transseptal & $\begin{array}{l}\text { AF, COPD, } \\
\text { decompensated } \\
\text { HF }\end{array}$ & 2: MVRep, MVR & 0.52 & 80 & 2 & 70 \\
\hline
\end{tabular}

AF, atrial fibrillation; AVR, aortic valve replacement; COPD, chronic obstructive pulmonary disease; EF, left ventricular ejection fraction; HF, heart failure; IDDM, insulin dependent diabetes mellitus; MVR, mitral valve replacement; MVRep, mitral valve surgical repair; NYHA, New York Heart Association; SPAP, systolic pulmonary artery pressure; STS PROM, Society of Thoracic Surgeons predicted risk of mortality; TR, tricuspid regurgitation. 
Table 2 Mitral valve characteristics.

\begin{tabular}{|c|c|c|c|c|c|c|c|c|c|c|c|c|}
\hline Patient \# & Valve type & Size $(\mathrm{mm})$ & ID (mm) & Failure mode & $\begin{array}{l}\text { Years after } \\
\text { MVR }\end{array}$ & $\begin{array}{l}\text { Measured ID } \\
(\mathrm{mm})\end{array}$ & THV type & Size & $\begin{array}{l}\text { Baseline VG } \\
(\mathrm{mm} \mathrm{Hg})\end{array}$ & $\begin{array}{l}\text { Final VG } \\
(\mathrm{mm} \mathrm{Hg})\end{array}$ & $\begin{array}{l}\text { Baseline MR } \\
\text { grade }\end{array}$ & $\begin{array}{l}\text { Final MR } \\
\text { grade }\end{array}$ \\
\hline 1 & CE, Porcine & 27 & 25 & $\begin{array}{l}\text { MR: reduced } \\
\text { leaflet mobility }\end{array}$ & $y^{9}$ & 22 & Sapien & 26 & 25 & 3 & 3 & TV 1 \\
\hline 2 & $\begin{array}{l}\text { Medtronic, } \\
\text { Hancock II }\end{array}$ & 25 & 22 & $\begin{array}{l}\text { MR: reduced } \\
\text { leaflet mobility }\end{array}$ & $\begin{array}{l}10 \\
y\end{array}$ & 21 & Sapien XT & 23 & 9 & 4 & 3 & 0 \\
\hline 3 & CE, Porcine & 27 & 25 & $\begin{array}{l}\text { MR: leaflet } \\
\text { prolapse }\end{array}$ & 7 & 25 & Sapien XT & 29 & 28 & 1 & 3 & 0 \\
\hline
\end{tabular}

The valves and their manufacturers are as follows: CE porcine (Carpentier-Edwards Porcine mitral valve, Edwards Lifesciences, Irvine, California); Sapien and Sapien XT (Edwards Lifesciences); Medtronic Hancock II mitral valve (Medtronic, Minneapolis, Minnesota); EOA, effective orifice area; ID, inner stent diameter according to manufacturers' specifications; measured ID, inner stent diameter according to intraprocedural transesophageal echocardiographic measurements; MR, mitral regurgitation; MVR, mitral valve replacement; THV, transcatheter heart valve; TV, transvalvular; VG, mean transvalvular gradient. 


\section{References}

1. Seiffert M, Conradi L, Baldus S, et al. Transcatheter mitral valve-in-valve implantation in patients with degenerated bioprostheses. J Am Coll Cardiol Interv. 2012;5:341-9.

2. Gopalamurugan AB, Pantazis A, Schievano S. Percutaneous transvenous mitral valve implantation. J Am Coll Cardiol. 2013;61:e143.

3. Webb J, Wood D, Ye J. Transcatheter valve-in-valve implantation for failed bioprosthetic heart valves. Circulation. 2010;121:1848-57.

4. Montorfano M, Latib A, Chieffo A. Successful percutaneous anterograde transcatheter valve-in-valve implantation in the mitral position. JACC Cardiovasc Interv. 2011;4:1246-7.

5. Michelena HI, Alli O, Cabalka AK, et al. Successful percutaneous transvenous antegrade mitral valve-invalve implantation. Catheter Cardiovasc Interv. 2013;81:E219-24.

6. Cullen MW, Cabalka AK, Alli OO, et al. Transvenous, antegrade melody valve-in-valve implantation for bioprosthetic mitral and tricuspid valve dysfunction: a case series in children and adults. JACC Cardiovasc Interv. 2013;6:598-605.

7. Merino JA, Alcántara MA, Escutia HH, et al. Transcatheter transapical mitral valve-in-valve implantation: case report. Rev Mex Cardiol. 2016;27:133-40.

8. Cheung A, Webb J, Wong DR, et al. Transapical transcatheter mitral valve-in-valve implantation in a human. Ann Thorac Surg. 2009;87:e18-20.

9. Cheung A, Webb J, Barbanti M, et al. 5-Year experience with transcatheter transapical mitral valve-in-valve implantation for bioprosthetic valve dysfunction. J Am Coll Cardiol. 2013;61:1759-66.
10. Dvir D, Webb J, Schafer U, et al. Transcatheter mitral valve-in-valve/valve-in-ring implantations for degenerative post-surgical valves: results from the global valve-in-valve registry. J Am Coll Cardiol. 2013;62(18_S1):B30.

José Alfredo Merino-Rajme ${ }^{a}$, Héctor Hugo

Escutia-Cuevas ${ }^{\mathrm{b}, *}$, Marco Antonio Alcántara-Meléndez ${ }^{\mathrm{a}}$, Ernesto Fernández-Ceseña ${ }^{a}$, Juán Francisco

García-García a , José Luis Fujigaki-Zaldívar ${ }^{\mathrm{b}}$, Julieta Danira Morales-Portano ${ }^{\mathrm{b}}$, Roberto Muratalla-González ${ }^{\mathrm{a}}$

${ }^{a}$ Hemodinámica y Cardiología Intervencionista, Centro Médico Nacional 20 de Noviembre, ISSSTE, Ciudad de México, Mexico

${ }^{\mathrm{b}}$ División de Cardiología, Centro Médico Nacional 20 de Noviembre, ISSSTE, Ciudad de México, Mexico

* Corresponding author at: Avenida Coyoacán 1617, Interior APH7, Colonia Del Valle Sur, Delegación Benito Juárez, Ciudad de México, Mexico. Tel.: +52 5539386401.

E-mail address: perseoyarista@hotmail.com (H.H. EscutiaCuevas).

https://doi.org/10.1016/j.acmx.2017.10.004 1405-9940/

(c) 2017 Instituto Nacional de Cardiología Ignacio Chávez. Published by Masson Doyma México S.A. This is an open access article under the CC BY-NC-ND license (http://creativecommons.org/licenses/ by-nc-nd/4.0/).

\section{¿Dónde está el tronco coronario izquierdo?}

\section{Where is the left main coronary artery?}

La incidencia de anomalías coronarias es baja en la población general, oscilando entre el $0.46-1.55 \%$, y la agenesia de tronco coronario izquierdo $(\mathrm{TCl})$ es una de las menos observadas ${ }^{1,2}$. Se trata de una entidad extremadamente rara en la que no existe el ostium coronario izquierdo y el TCI termina ciegamente ${ }^{3}$. De los casos publicados, el $50 \%$ afectan a la edad pediátrica, y entre ellos, el $30 \%$ se asocia a otras anomalías coronarias ${ }^{4}$. Puede aparecer aisladamente, como en los pacientes que aquí mostramos, o asociada a otras enfermedades, como la homocistinuria, la ataxia de Friedreich, el síndrome de Hurler, la progeria y el síndrome rubeólico ${ }^{5}$.

Se presentan 2 casos clínicos representativos de esta enfermedad de baja prevalencia en la población general.

El primer caso se trata de un varón de 59 años con hipercolesterolemia y extabaquismo como factores de riesgo cardiovascular. Es derivado a nuestro centro para la realización de una coronariografía por angina de esfuerzo, con ergometría clínicamente negativa y eléctricamente positiva con descenso del segmento ST de $2 \mathrm{~mm}$ en el tercer estadio del protocolo de Bruce. El cateterismo muestra una agenesia del $\mathrm{TCl}$ con visualización de un vaso hipoplásico submilimétrico. La arteria descendente anterior se visualiza a través de la arteria coronaria anómala, que nace de la rama marginal aguda precoz desde el segmento proximal de la coronaria derecha (CD). El trayecto anómalo presenta efecto kinking y compresión sistólica. La circunfleja se visualiza a través de la arteria conal, con salida independiente en cañón de escopeta ligeramente craneal al ostium de la $\mathrm{CD}$, con trayecto anómalo y efecto kinking sin evidente efecto milking. La CD es dominante, de gran calibre y sin estenosis angiográficamente significativas (figs. 1 y 2).

El segundo caso se trata de un paciente varón de 58 años con antecedentes de cardiopatía isquémica familiar precoz, hipertensión arterial y tabaquismo activo. Ingresa por síndrome coronario agudo sin elevación del segmento ST de alto riesgo, con isquemia subepicárdica inferior en el electrocardiograma y elevación de marcadores de necrosis miocárdica (TnT US $272.3 \mathrm{ng} / \mathrm{L}$ ). Se realiza un ecocardiograma que muestra un ventrículo izquierdo no dilatado con función sistólica conservada y sin anomalías regionales en la contractilidad. No se observaron hallazgos patológicos a nivel valvular ni pericárdico. Se realiza una coronariografía que muestra origen anómalo de la coronaria izquierda con salida independiente de la descendente anterior y circunfleja desde el seno de Valsalva derecho. La CD es un vaso dominante de gran calibre y desarrollo con irregularidades en los segmentos medio y distal, sin estenosis angiográficamente significativas. La descendente posterior es un vaso de gran desarrollo que presenta una estenosis crítica por placa complicada, sobre la que se realiza intervencionismo coronario percutáneo con angioplastia con balón e implante de un stent farmacoactivo liberador de sirolimus, con buen resultado angiográfico final y flujo TIMI 3 (fig. 3). 